\title{
A l'aide! Comment puis-je trouver un successeur?
}

\section{Remo Osterwalder}

Dr, membre du Comité central de la FMH, responsable du département Médecins en libre-pratique

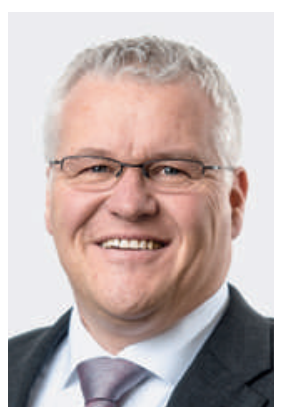

Si trouver un successeur était autrefois une formalité, cela relève aujourd'hui toujours plus du parcours du combattant pour de nombreux médecins de famille mais aussi pour certains spécialistes, qui mettent parfois plusieurs années avant de pouvoir remettre leur cabinet. Comment en sommes-nous arrivés là? Confrontée d'une part à l'évolution des attentes de la jeune génération, la profession de médecin doit également composer avec les débats incessants autour de la maîtrise des coûts et l'augmentation de la bureaucratie, qui ne rendent pas toujours service au plus beau métier du monde. Or à mesure que la population vieillit, le besoin de relève se fait toujours plus pressant et le nombre de médecins formés en Suisse ne suffit plus. De plus, le prix lié à la vente d'un cabinet, tel qu'il se pratiquait autrefois, n'est plus exigé de manière systématique, et peut parfois même devenir un obstacle à la vente. Certains confrères ont cependant su tirer leur épingle du jeu en devenant formateurs au cabinet avant de partir à la retraite, une très bonne expérience qui leur a permis à eux ainsi qu'aux médecins en formation de nouer des contacts intéressants, mais aussi de trouver un nouvel intérêt à investir dans l'infrastructure de leur cabinet. Et parfois même de trouver un successeur.

\section{Les cabinets formateurs disposent d'un avan- tage décisif.}

Qu'est-ce qui rend un cabinet intéressant? La question de l'emplacement n'est pas seulement importante en périphérie, mais aussi et surtout là où il existe une proximité avec d'autres confrères. Pour être intéressant, un emplacement doit répondre à plusieurs critères: le cabinet est-il proche du centre ou de la gare? Dispose-t-il d'un nombre suffisant de places de parc? Est-il bien desservi par les transports publics? Existet-il des possibilités d'extension dans le cas où le médecin souhaiterait partager ses locaux avec d'autres confrères? La taille du cabinet correspond-elle au nombre de patients par jour, sachant que des locaux trop exigus sont susceptibles de générer du stress, d'avoir un impact négatif sur la satisfaction au travail voire de contribuer à une fluctuation élevée du personnel? Très souvent, le point de vue des patients n'est pas ou pas suffisamment pris en compte dans le choix des locaux. Or certains aspects qui nous semblent pratiques à nous peuvent s'avérer problématiques pour les patients, raison pour laquelle il peut être utile de consulter des organisations de patients comme Pro Senectute ou des associations de défense des intérêts des personnes handicapées.

\section{Il ne faut pas négliger les investissements même après plusieurs années d'activité.}

Une autre question essentielle est celle de savoir si le cabinet dispose d'un système informatique. En effet, la plupart de nos jeunes confrères sont habitués à avoir constamment sur eux une tablette ou un iPad lors des consultations à l'hôpital. De plus, pouvoir accéder à distance au système informatique du cabinet peut s'avérer utile en cas d'urgence, mais aussi lors de visites à domicile. Si devenir formateur n'est pas possible pour des raisons de temps disponible ou parce que l'heure de la retraite approche à grands pas, je ne peux que conseiller aux collègues concernés de se mettre en contact avec des confrères qui proposent des places de formation, en s'adressant à leur société médicale cantonale ou aux autorités sanitaires de leur canton qui pourront leur fournir les coordonnées des formateurs. De plus en plus de projets visant à assurer la pérennité des soins ambulatoires voient le jour, à l'instar des forums d'échange d'informations et de contacts présents dans certains cantons. Si vous décidez de faire appel à un architecte ou à d'autres spécialistes, n'oubliez pas non plus d'étudier leurs références avec le plus grand soin. Cela vous permettra d'économiser de l'argent mais également de vous épargner certains ennuis. 